\title{
Cyclosporin A Has Direct Effects on Adult Neural Precursor Cells
}

\author{
Jessica Hunt, ${ }^{1}$ Allan Cheng, ${ }^{1}$ Amy Hoyles, ${ }^{1}$ Eric Jervis, ${ }^{2}$ and Cindi M. Morshead ${ }^{1}$ \\ ${ }^{1}$ Department of Surgery, Donnelly Centre for Cellular and Biomolecular Research, University of Toronto, Toronto, Ontario M5S 3E1, Canada, and \\ ${ }^{2}$ Department of Chemical Engineering, University of Waterloo, Waterloo, Ontario N2L 3G1, Canada
}

\begin{abstract}
Multipotent, self-renewing neural stem cells and their progeny [collectively referred to as neural precursor cells (NPCs)] represent a population of cells with great promise for CNS repair. To effectively harness their potential for therapeutic applications, the factors that regulate NPC behavior and/or fate must be well understood. The ability of immunomodulatory molecules to affect NPC behavior is of interest because of recent work elucidating the complex interactions between the immune system and nervous system. Herein, we examined the effects of cyclosporin A, a commonly used immunosuppressive molecule, on NPC proliferation kinetics, survival, and fate using in vitro assays at the population level and at the single-cell level. The use of pure populations of NPCs revealed a direct effect of cyclosporin A on cell survival, resulting in increased numbers and larger colonies, with no effect on proliferation kinetics. Cyclosporin A did not alter the differentiation profile of NPC colonies, indicating that it did not promote selective survival of a particular neural lineage. Additionally, we observed decreased cell-cell adhesions in developing cyclosporin A-treated NPC colonies. Consistent with the in vitro observations, in vivo administration of cyclosporin A to adult animals increased the numbers of NPCs within the neurogenic niche lining the lateral ventricles. Together, our findings establish that cyclosporin A has direct effects on NPCs both in vitro and in vivo, making it a promising candidate molecule for developing clinically relevant strategies to stimulate NPCs for brain repair.
\end{abstract}

\section{Introduction}

Neural precursor cells (NPCs) are excellent candidates for developing therapeutic strategies to repair the injured CNS (Erlandsson and Morshead, 2006). Neural stem cells reside in the subependyma of the forebrain lateral ventricles, where they divide to give rise to progeny that generate neuroblasts that migrate to the olfactory bulb and differentiate into interneurons (Lois and Alvarez-Buylla, 1994; Morshead et al., 1994). They can be isolated in vitro in the presence of growth factors, whereby single cells proliferate to form free-floating colonies termed "neurospheres" (Reynolds and Weiss, 1992). The stem and progenitor cells that make up the neurospheres display the cardinal stem cell properties of self-renewal and multipotency. The inherent capacity of NPCs to proliferate, migrate, and differentiate in vivo, along with the capacity for in vitro expansion, makes them excellent targets for neural repair strategies and has led to much interest in identifying extrinsic cues and signaling pathways that regulate their kinetics and fate.

Recent work showing that NPC proliferation, migration, and differentiation profiles can change in response to inflammation and degenerative damage has led to an interest in the effects of

Received Dec. 3, 2009; accepted Jan. 5, 2010.

This work was funded by the Stem Cell Network, the Heart and Stroke Foundation, and Canadian Institutes of Health Research. We thank Darik Gamble and Richard Benko at the University of Waterloo for their technical assistance.

Correspondence should be addressed to Cindi M. Morshead, University of Toronto, Donnelly Centre for Cellular and Biomolecular Research, Room 1006, 160 College Street, Toronto, 0N M5S3E1, Canada. E-mail: cindi.morshead@ utoronto.ca.

D0I:10.1523/JNEUROSCI.5991-09.2010

Copyright $\odot 2010$ the authors $\quad 0270-6474 / 10 / 302888-09 \$ 15.00 / 0$ immunomodulatory molecules on NPC behavior (Butovsky et al., 2006; Pluchino and Martino, 2008). NPCs express several immune-relevant molecules that enable them to functionally interact with the inflammatory microenvironment such as cell adhesion molecules, integrins, and chemokine receptors (Ben-Hur et al., 2003, 2006). What remains largely unknown is how immunomodulatory molecules change NPC behavior and whether these effects are due to direct or indirect actions on NPCs.

Cyclosporin A is an immunosuppressive drug used clinically to treat autoimmune disorders and prevent graft rejection following organ transplant (Borel et al., 1976). We asked whether this immunomodulatory molecule can influence NPC behavior. Cyclosporin A crosses the plasma membrane, binds, and blocks a family of peptidyl-prolyl isomerases known as cyclophilins (Handschumacher et al., 1984; Fischer et al., 1989; Takahashi et al., 1989). Cyclophilins are distributed in various cellular organelles where they facilitate protein folding, act as chaperones, and play a role in cell signaling (Wang and Heitman, 2005). Cyclosporin A exerts its immunosuppressive effect by binding to cyclophilin A and the drug-receptor complex inhibits the dephosphorylase activity of calcineurin. Blocking calcineurin inhibits the translocation of nuclear factor of activated T-cells from the cytosol into the nucleus, thus preventing transcription of interleukin-2, a cytokine that induces T-cell activation and proliferation (Kay et al., 1983; Flanagan et al., 1991; Fruman et al., 1992). It is unknown whether cyclosporin A can affect NPCs and if so, whether this pathway is involved.

Herein, we use in vitro and in vivo assays to examine the effects of cyclosporin A on NPC behavior. First, we demonstrate that cyclosporin A acts directly on NPCs to enhance their survival and 
modify cell-cell adhesion. Further, we show that the administration of cyclosporin A to adult animals, in the absence of injury, leads to increased numbers of neural stem cells in vivo. The selective effect of cyclosporin A on NPC survival suggests that cyclosporin A is a promising molecule for modulating NPCs.

\section{Materials and Methods}

Neurosphere assay. Neural stem cells were isolated by dissection of the forebrain subependyma of adult male CD1 mice (6-8 weeks old, 25-30 g; Charles River) as previously described (Morshead et al., 2003). Briefly, tissue was digested with enzymes $(1.33 \mathrm{mg} / \mathrm{ml}$ trypsin, $0.67 \mathrm{mg} / \mathrm{ml}$ hyaluronidase, and $0.2 \mathrm{mg} / \mathrm{ml}$ kynurenic acid; all from Sigma-Aldrich) for 40 $\mathrm{min}$ at $37^{\circ} \mathrm{C}$. Enzyme activity was inhibited with $0.67 \mathrm{mg} / \mathrm{ml}$ trypsin inhibitor (Roche Diagnostics), and the tissue was mechanically dissociated into a single-cell suspension. Cells were plated at clonal density (5-10 cells/ $\mu \mathrm{l}$ ) (Coles-Takabe et al., 2008) in 24-well polystyrene plates (VWR Scientific) with serum-free medium (SFM) supplemented with epidermal growth factor (20 ng/ml; Sigma-Aldrich), basic fibroblast growth factor (10 ng/ml; Sigma-Aldrich), heparin (7.35 ng/ml; SigmaAldrich), and 1\% penicillin/streptomycin (Invitrogen). Stock solution of cyclosporin A ( $0.2 \mathrm{mg} / \mathrm{ml}$; Bioshop Canada) was made by dissolving solid cyclosporin $\mathrm{A}$ in a 1:1 anhydrous ethyl alcohol:growth factorsupplemented SFM solution and subsequently added to the cultures at various concentrations. For long-term experiments, bulk cell cultures were passaged every $7 \mathrm{~d}$ for up to 6 weeks ( $\mathrm{P} 5$ neurospheres) by pelleting the sample and mechanically dissociating the pellet using a small borehole pipet and growth factor-supplemented SFM. Cells were exposed to cyclosporin A $(100 \mathrm{ng} / \mathrm{ml})$ at various times after the initial plating. To assay for neural stem cell survival and self-renewal, single neurospheres from cyclosporin A-treated and untreated conditions were collected, triturated into a single-cell suspension, and plated ( 1 sphere/well) in growth factor-supplemented SFM. For differentiation assays, individual neurospheres were collected from each condition, gently triturated, and plated onto Matrigel-coated wells in the presence of $10 \%$ FBS for $7 \mathrm{~d}$.

Live cell imaging. Neurospheres were dissociated to create a single-cell suspension, and the cells were loaded into a custom-designed culture chamber referred to as a "gap chamber" (Ramunas et al., 2006). The chamber is formed by a glass slide and glass coverslip that is supported by $4.5 \mu \mathrm{m}$ diameter polystyrene beads (Polysciences). The chamber confines NPCs to a monolayer such that cell divisions occur side-by-side and each cell is visible throughout colony formation. The chambers were filled with growth factor-supplemented SFM with or without cyclosporin $\mathrm{A}$, and they were incubated at $37^{\circ} \mathrm{C}$ with $5 \% \mathrm{CO}_{2}$ humidified air. Images were captured at $20 \times$ or $40 \times$ magnification every 3 min using a digital camera (XCD-SX910; Sony) and inverted microscope (Axiovert 200; Carl Zeiss). Cells were imaged for 3 or $8 \mathrm{~d}$ and colonies subsequently fixed with $4 \%$ paraformaldehyde (Sigma-Aldrich) and stained. In the $8 \mathrm{~d}$ imaging experiments, colonies were differentiated by adding $10 \%$ fetal bovine serum (FBS; HyClone) to the chambers on day 5. Images were imported into custom-developed software that allows for tracking of cells, generation of lineage trees, and storage of cell behavior/fate data (Cell Hunter).

Semiquantitative reverse transcription-PCR. Total RNA was isolated using a RNeasy Mini Kit (Qiagen), precipitated in ethanol, and treated with DNase I (Invitrogen) before resuspension in $12 \mu \mathrm{l}$ of RNase-free water. Reverse transcription (RT) and PCR were performed sequentially in the same tube on equal amounts of isolated RNA using a OneStep RT-PCR kit (Qiagen). The primers used for PCR amplification were as follows: cyclophilin A forward primer, 5'-GTC TCC TTC GAG CTG TTT GC- $3^{\prime}$, and cyclophilin A reverse primer, $5^{\prime}$-ATC TTC TTG CTG GTC TTG CC-3'; cyclophilin B forward primer, 5' -GGC TCC GTC GTC TTC CTT TT- $3^{\prime}$, and cyclophilin B reverse primer, $5^{\prime}$-ACT CGT CCT ACA GAT TCA TCT CC-3'; cyclophilin C forward primer, $5^{\prime}$-GAG CCC TGG TGT CTT CTT CG-3', and cylophilin C reverse primer, 5'-GGG ACC TCA ACC ACA AAG GG-3'; cyclophilin D forward primer, 5' -CTT CCA CAG GGT GAT CCC AG-3', and cyclophilin D reverse primer, 5'-ACT GAG AGC CAT TGG TGT TGG-3'; calcineurin forward primer, 5'-GTG AAA GCC GTT CCA TTT CCA-3', and calcineurin reverse primer, 5'-GAA TCG AAG CAC CCT CTG TTA TT-3'; interleukin-2 forward primer, 5'-TGC TCC TTG TCA ACA GCG-3'; and interleukin-2 reverse primer, 5' -TCA TCA TCG AAT TGG CAC TC-3'; CD98 forward primer, $5^{\prime}$-TGA TGA ATG CAC CCT TGT ACT TG-3', and CD98 reverse primer, 5'-GCT CCC CAG TGA AAG TGG A-3'; CD147 forward primer, 5'-GGC TGG TTT CCT CAA GGC A-3', and CD147 reverse primer, 5' -TAG GCG GCA TGG ATG TGA AC-3' (sequence based on BLAST search for mRNA NM_001077184); $\beta$-actin forward primer, 5'-AGG CCA ACC GTG AAA AGA TGA C-3', and $\beta$-actin reverse primer, $5^{\prime}$-GTA CAT GGT GGT ACC ACC AGA C-3 (all from Sigma Genosys). After 40 cycles of amplification, $10 \mu \mathrm{l}$ of the reaction mix was electrophoresed on $2 \%$ agarose gels and stained with SYBRsafe DNA gel stain (Invitrogen).

Stimulating interleukin-2 production. Splenic tissue was collected from adult male CD1 mice. Tissue was dissociated to create a single-cell suspension using a 25 gauge needle, $1 \mathrm{ml}$ syringe, and RPMI 1640 medium (Sigma-Aldrich). Cells were plated at 5000 cells/ $\mu$ l in T25 falcon flasks (Becton Dickinson) in RPMI 1640 medium supplemented with 10\% FBS (HyClone), 1\% penicillin/streptomycin (Invitrogen), 20 mM HEPES, 200 mM L-glutamine, and $5 \mu \mathrm{l} / \mathrm{ml}$ concanavalin A (all three from SigmaAldrich). The cultures were incubated at $37^{\circ} \mathrm{C}$ for $20 \mathrm{~h}$ before RNA isolation and detection. Controls included the addition of $5 \mu \mathrm{l} / \mathrm{ml}$ concanavalin A to neurosphere culture medium as described above.

In vivo studies. Adult male CD1 mice (6-8 wks, 25-30 g; Charles River) were housed in the University of Toronto animal facilities and maintained in accordance with the institutional guidelines. Animals were immunosuppressed with a daily dose of cyclosporin A $(15 \mathrm{mg} / \mathrm{kg} / \mathrm{d})$ delivered subcutaneously or intraventricularly via an osmotic minipump (Alzet Osmotic Pumps) ( $n=4$ animals/cyclosporin A or saline/s.c. or i.v. infusion). The pump delivered cyclosporin A or saline at a rate of $0.5 \mu \mathrm{l}$ per $\mathrm{h}$ for $14 \mathrm{~d}$. Neural stem cells were isolated by dissection of the forebrain subependyma as described above. The cells were plated at a density of 5 cells/ $\mu$ l in growth factor-supplemented SFM, and the neurospheres that formed were counted on day 7 of culture. A separate group of cyclosporin A- or saline-treated animals ( $n=4$ per group) received four BrdU injections (s.c., one every $2.5 \mathrm{~h}, 60 \mathrm{mg} / \mathrm{kg}$ ) and were killed $1 \mathrm{~h}$ after the last injection. Animals were perfused with $4 \%$ paraformaldehyde and the brains were removed and cryoprotected overnight in 20\% sucrose (Sigma-Aldrich) at $4^{\circ} \mathrm{C}$. Cryostat sections $(17 \mu \mathrm{m})$ were mounted on Superfrost slides (Fisher Scientific) and stained for BrdU or DAPI as described below.

Immunohistochemistry. Cell colonies (neurospheres or colonies in the gap chambers) were fixed in $4 \%$ paraformaldehyde for $20 \mathrm{~min}$ at room temperature and then washed (all washes were done three times, $5 \mathrm{~min} /$ rinse, at $37^{\circ} \mathrm{C}$ using $1 \times$ Stockholm's PBS). Colonies were permeabilized using $0.3 \%$ Triton X-100 (Sigma-Aldrich) for $20 \mathrm{~min}$, rinsed, then blocked with $10 \%$ normal goat serum (NGS; Jackson Laboratories) for $1 \mathrm{~h}$ at $37^{\circ} \mathrm{C}$. All antibodies were diluted in $1 \times$ Stockholm's PBS plus $1 \%$ NGS. Fixed clones were incubated with primary antibodies overnight at $4^{\circ} \mathrm{C}$, washed, incubated with an appropriate secondary antibody for $2 \mathrm{~h}$ at $37^{\circ} \mathrm{C}$, washed, and then fluorescently imaged using an Axiovert 200 (Carl Zeiss). Undifferentiated colonies were stained for Nestin (mouse monoclonal IgG, 1:500; Millipore Bioscience Research Reagents). Differentiated colonies were stained for O4 (mouse monoclonal IgM, 1:75; Millipore Bioscience Research Reagents), $\beta$ III tubulin (mouse monoclonal IgG, 1:500; Sigma-Aldrich), MAP-2 (mouse monoclonal IgG, 1:1000; Millipore Bioscience Research Reagents), and GFAP (rabbit polyclonal IgG, 1:500; Abcam). The secondary antibodies were as follows: Alexa Fluor 488 goat anti-mouse IgG; Alexa Fluor 568 goat anti-mouse IgM; Alexa Fluor 350 goat anti-rabbit IgG (all 1:300; Invitrogen). Cell nuclei were counterstained with Hoechst 33342 nuclear stain $(0.725 \mu \mathrm{g} / \mathrm{ml}$; Invitrogen) for $20 \mathrm{~min}$ at $37^{\circ} \mathrm{C}$ and then washed. Brain sections mounted on slides were treated with $2 \mathrm{~m}$ hydrochloric acid (Sigma-Aldrich) for 20 $\min$ at $37^{\circ} \mathrm{C}$, washed, and incubated with BrdU antibody (rat monoclonal IgG, 1:100; Abcam) overnight at $4^{\circ} \mathrm{C}$. Slides were washed, incubated with Alexa Fluor 568 goat anti-rat IgG for $2 \mathrm{~h}$ at $37^{\circ} \mathrm{C}$, washed, and then fluorescently imaged. For quantification of the numbers of Hoechstlabeled cells in vitro, positive cells were counted using a $5 \times$ objective, counting all cells within the field of view in five areas of the well [top, 
bottom, left side, right side (all equal distance from the edge of the well) and center]. An estimate of the total cells in a well was made using the area of the well $\left(2.0 \mathrm{~cm}^{2}\right)$, area of the field of view $\left(0.03 \mathrm{~cm}^{2}\right)$, and the average number of cells per field of view. For differentiated neurospheres, immunopositive cells were counted using a $20 \times$ objective from 10 spheres, 200 cells/sphere. For the gap chamber colonies, all cells were counted. Cell counts were from brain sections anterior to the crossing of the anterior commissure and caudal to the crossing of the corpus callosum. Adjacent sections were stained with Vectashield mounting medium with DAPI (Vector Laboratories) for total cell counts. The numbers of labeled cells $\left(\mathrm{BrdU}^{+}\right.$ or $\mathrm{DAPI}^{+}$) were counted and averaged from three fields of view from three sections per animal at $20 \times$ magnification in each of the dorsolateral corner and the lateral wall of the lateral ventricle.

Statistical methods. Statistical analysis was performed using the Student's $t$ test for twogroup comparisons and ANOVA for multiple group comparisons (SigmaStat statistical package). All data are reported as means \pm SEM.

\section{Results}

\section{Cyclosporin A enhances neurosphere} numbers and size

We first looked for the presence of cyclophilins and calcineurin in NPCs to determine whether these cells have the intracellular machinery necessary to respond to cyclosporin A. Cyclophilins are a family of proteins that bind cyclosporin A (Guo et al., 2001). They are greatly enriched in the brain (as shown using immunohistochemistry, in situ hybridization, and autoradiography) at concentrations 10-50 times higher than concentrations in immune cells. Calcineurin, the diphosphatase upstream of interleukin-2 production, is also greatly enriched in the brain (Dawson et al., 1994). We used RT-PCR on adult-derived neurospheres, which comprise a pure population of NPCs, and looked for the presence of the cyclophilins known to have a high affinity for cyclosporin A, namely cyclophilins A, B, C, and D (Hamilton and Steiner, 1998), as well as calcineurin, which is the predominant protein inhibited by the cyclosporin A-cyclophilin A complex (Liu et al., 1991). We detected cyclophilins A, B, C, and D as well as calcineurin within NPCs with relative levels of cyclophilin A being the greatest (Fig.

$1 A)$. Thus, NPCs can interact with cyclosporin A and the presence of multiple cyclophilins suggests that more than one signaling pathway could be activated in the presence of the drug.

We next examined the affect(s) of cyclosporin A on NPC behavior at the population level using the neurosphere assay. Neurospheres represent a mixed population of stem and progenitor cells. Stem cells comprise a rare subpopulation $(<1 \%)$ while the progenitor cells comprise the vast majority of cells (Morshead

C

D
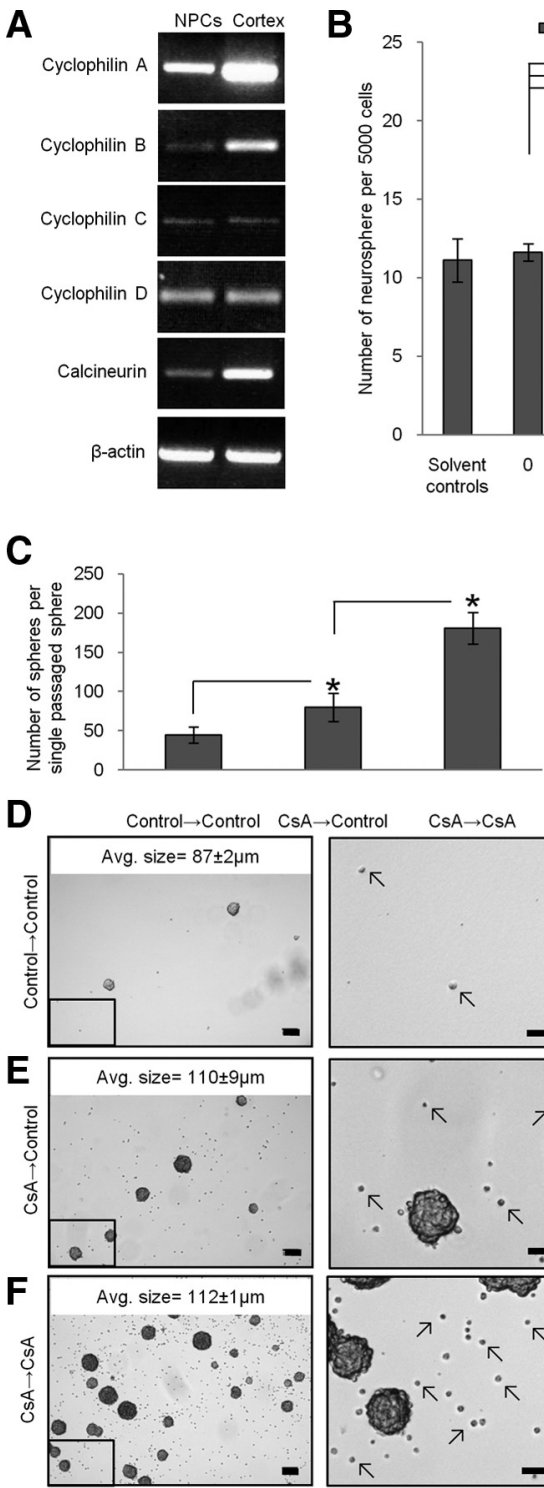

Figure 1. Cyclosporin $A$ increased neurosphere numbers and size. $A, R T-P C R$ confirmed the presence of cyclophilins $A, B, C$, and $D$ and calcineurin in P1 neurosphere-derived cells. $B$, Cyclosporin A was added to subependyma-derived (primary) cells and P1 neurosphere-derived cells ( $n=4$ trials each, 6 wells/trial). For subependyma-derived cells (black bars), neurosphere numbers increased 1.7-fold at $100 \mathrm{ng} / \mathrm{ml}$ cyclosporin A and 1.4-fold at $500 \mathrm{ng} / \mathrm{ml}$ cyclosporin A. For P1 neurosphere-derived cells (gray bars), a similar 1.8-fold increase in neurosphere numbers was observed at $100 \mathrm{ng} / \mathrm{ml}$ cyclosporin A. C, Control and cyclosporin A-treated P0 neurospheres were single-sphere passaged into control or cyclosporin A (CsA) conditions. We observed a 1.8-fold increase in numbers of $\mathrm{P} 1$ neurospheres that had been isolated in cyclosporin A. NPCs exposed to cyclosporin $A$ twice $(C s A \rightarrow C s A)$ produced 2.3-fold more neurospheres than NPCs exposed to cyclosporin A once. Examples of each condition are shown in $\boldsymbol{D}-\boldsymbol{F}$. A region (black box) of each image is enlarged to represent the live cells (arrows) that we typically observed in the different conditions (scale bar, $100 \mu \mathrm{m}$ ) (average size data represent mean \pm SEM for neurospheres in each condition). G-J, Primary (P0) dissected tissue and passaged neurospheres (P1-P5) were exposed to cyclosporin A (CSA) at various times and the numbers of neurospheres were assayed. Exposure to cyclosporin $A$ led to significantly more neurospheres relative to untreated controls $(0)$, regardless of the passage number. This effect is lost after one or two passages in the absence of cyclosporin A. ${ }^{*} p<0.05$; ${ }^{* *} p<0.01$. P0, Subependyma-derived cells; P1-P5 neurosphere-derived cells 1-5 weeks old. et al., 2002). Accordingly, the total number of neurospheres reflects the number of stem cells, while the size of an individual neurosphere reflects the number of progenitors that make up the bulk of a neurosphere. We established neurosphere cultures from adult brains using the standard conditions (epidermal growth factor plus fibroblast growth factor) in the presence or absence of cyclosporin A (0-5000 ng/ml). Cyclosporin A is dissolved in ethanol; hence, appropriate solvent controls were run for each concentration. After $7 \mathrm{~d}$ in culture, the numbers and size of 
neurospheres from each condition were assayed. We observed significant increases in neurosphere numbers at $100 \mathrm{ng} / \mathrm{ml}$ cyclosporin A ( $19 \pm 2$ spheres; $p<0.01 ; 1.7$-fold increase) and 500 $\mathrm{ng} / \mathrm{ml}$ cyclosporin A ( $16 \pm 1$ spheres; $p<0.05 ; 1.4$-fold increase) relative to $0 \mathrm{ng} / \mathrm{ml}$ cyclosporin $\mathrm{A}(12 \pm 1$ spheres) (Fig. $1 B)$. Further, we found that exposure to $100 \mathrm{ng} / \mathrm{ml}$ cyclosporin A produced significantly larger neurospheres on average compared with $0 \mathrm{ng} / \mathrm{ml}$ cyclosporin A ( $94 \pm 3 \mu \mathrm{m}$ vs $77 \pm 3 \mu \mathrm{m}$ diameters, respectively; $p<0.01)$. Neurosphere numbers and size significantly decreased at $5000 \mathrm{ng} / \mathrm{ml}$ cyclosporin A relative to $0 \mathrm{ng} / \mathrm{ml}$ cyclosporin A, suggesting that this high concentration was toxic to NPCs $(4 \pm 1.4$ spheres and $59 \pm 3 \mu \mathrm{m}$ diameter; $p<0.01$, both). No change in neurosphere numbers and/or size was observed in the solvent controls compared with $0 \mathrm{ng} / \mathrm{ml}$ cyclosporin A; thus, the solvent control data were combined (Fig. $1 B$ ). The increased numbers and size of neurospheres suggest that cyclosporin A has effects on both stem and progenitor populations, respectively.

Since the initially plated cells in cultures derived from adult brain dissections represent a mixed population of NPCs and mature brain derived cells (i.e., neural, endothelial, and blood cells) the increased numbers and size of neurospheres could be due to an indirect effect of cyclosporin A on these contaminating cells that subsequently modify the behavior of NPCs. To eliminate this possibility, we established cultures of pure populations of NPCs and exposed them to cyclosporin A. Control neurospheres (i.e., previously unexposed to cyclosporin A) were collected, dissociated into single cells, and plated in growth factor-supplemented medium with or without $100 \mathrm{ng} / \mathrm{ml}$ cyclosporin A. The numbers and size of neurospheres were assayed $7 \mathrm{~d}$ later. Identical to primary dissected tissue, cyclosporin A treatment resulted in a 1.8-fold increase in numbers of neurospheres $(21 \pm 1$ spheres vs $12 \pm 1$ spheres, treatment vs control; $p<0.01$ ) (Fig. $1 B$ ). In addition, we observed a significant increase in the average neurosphere diameter (90 \pm 12 vs $73 \pm 7 \mu \mathrm{m}$, treatment vs control; $p<0.05$ ). Hence, the observed effects of cyclosporin A are due to direct actions on NPCs. We further examined the effects of cyclosporin A on passaged cells by varying the time of exposure following the initial plating. Neurospheres were passaged for up to 6 weeks (P5 neurospheres) in culture, in the presence or absence of cyclosporin A (Fig. $1 G-K$ ). Regardless of the time spent in culture (in control conditions) the magnitude of the response to the initial exposure to cyclosporin A was identical to the response seen in primary cells cultured in cyclosporin A (1.8-fold increase). Moreover, neurosphere numbers return to control values when cyclosporin A is removed from the cultures within one or two passages. Hence, these findings support the hypothesis that cyclosporin A is having direct effects on NPCs.

The increased numbers of neurospheres in the presence of cyclosporin A can be explained by enhanced cell survival. Indeed, when individual neurospheres derived in the presence or absence of cyclosporin A were passaged into control conditions (no cyclosporin A), we observed the same 1.8-fold increase in the numbers of secondary neurospheres from the treated neurospheres $(80 \pm 24$ spheres vs $45 \pm 12$ spheres, cyclosporin A to control vs control; $p<0.05$ ) (Fig. 1C), revealing that an individual neurosphere grown in the presence of cyclosporin A contains more stem cells. Strikingly, individual neurospheres grown in cyclosporin A and passaged into cyclosporin A gave rise to a further 2.3 -fold increase in the numbers of secondary neurospheres (181 \pm 20 spheres vs $80 \pm 24$ spheres, cyclosporin A to cyclosporin A vs cyclosporin A to control; $p<0.05$ ). As well, the average diameter of the cyclosporin A-exposed secondary neuro- spheres was significantly larger than control (no cyclosporin A) neurospheres $(112 \pm 1 \mu \mathrm{m}$ vs $87 \pm 2 \mu \mathrm{m}$, cyclosporin A to cyclosporin A vs control; $p<0.01$ ) (Fig. $1 D-F$ ). In addition to more numerous and larger neurospheres, there were many more live cells on the bottom of cyclosporin A-treated wells (Fig. $1 D-$ $F$ ). To quantify this observation, we counted the number of Hoechst-labeled cells on the bottoms of control and cyclosporin A-treated culture wells after $7 \mathrm{~d}$. We observed a significant fivefold increase in numbers of Hoechst-labeled cells in cyclosporin A treatment $(1030 \pm 182$ cells/well vs $208 \pm 14$ cells/well, treatment vs control; $p>0.01)$. The increased numbers of cells was further confirmed using the PicoGreen assay for viable cells in which we observed significantly greater numbers of cells in the presence of cyclosporin A (data not shown). The increased numbers and size of neurospheres are consistent with cyclosporin $\mathrm{A}$ increasing both neural stem and progenitor cell survival; however, the possibility of cyclosporin A altering the rate of proliferation of progenitor cells (thereby, increasing the size of the neurospheres) could not be ruled out.

\section{Live cell imaging analysis reveals that cyclosporin A enhances NPC survival}

To distinguish between effects of cyclosporin A on survival versus effects on proliferation kinetics, we examined NPC colony formation at the single-cell level using live cell imaging. Cells were placed in custom designed chambers called gap chambers which enable imaging of individual cells during colony formation. The gap chamber confines NPCs to a monolayer such that divisions occur side-by-side to form 2-dimensional colonies in which each cell can be tracked over the entire experiment (Ramunas et al., 2006) (see supplemental Movie S1, available at www.jneurosci. org as supplemental material). Neurosphere-derived NPCs were loaded into gap chambers containing growth factor-supplemented medium with $100 \mathrm{ng} / \mathrm{ml}$ cyclosporin A (treated group) or $0 \mathrm{ng} / \mathrm{ml}$ cyclosporin A (untreated group). Images were collected at $20 \times$ magnification every $3 \mathrm{~min}$ for a period of $3 \mathrm{~d}$, at which time the colonies were fixed, stained with Hoechst (nuclear stain), and stained for Nestin expression (a marker of NPCs). The images were converted into movies and lineage analysis was performed. In total, 132 untreated NPCs and 139 cyclosporin A-treated NPCs were imaged within chambers and tracked for $3 \mathrm{~d}$ from three independent trials.

Based on the observations from the neurosphere assay, we hypothesized that cyclosporin A enhanced NPC survival. Accordingly, we examined the amount of cell death using the live cell imaging analysis and quantifying the numbers of cells that died within the chambers in control and treatment conditions, and found significantly less cell death at all times over the $3 \mathrm{~d}$ period within the cyclosporin A-treated group (Fig. 2 A). By day $3,31 \pm 2 \%$ of the total numbers of NPCs died in control conditions versus $16 \pm 3 \%$ in cyclosporin A-treated cultures $(p<$ $0.05)$. This increase in cell survival was not concomitant with increased proliferation of the surviving cells as the numbers of plated NPCs to undergo at least one cell division over the $3 \mathrm{~d}$ period did not differ between the groups $(60 \pm 14 \%$ vs $57 \pm$ $19 \%$, treatment vs control) (Fig. 2B). Hence, in cyclosporin A-treated cultures, greater numbers of cells survive and go on to form colonies.

\section{Cyclosporin A increases colony size by enhancing the survival} of NPCs within clones

Similar to the neurosphere analysis, we observed a significant increase in the size of cyclosporin A-treated colonies compared 


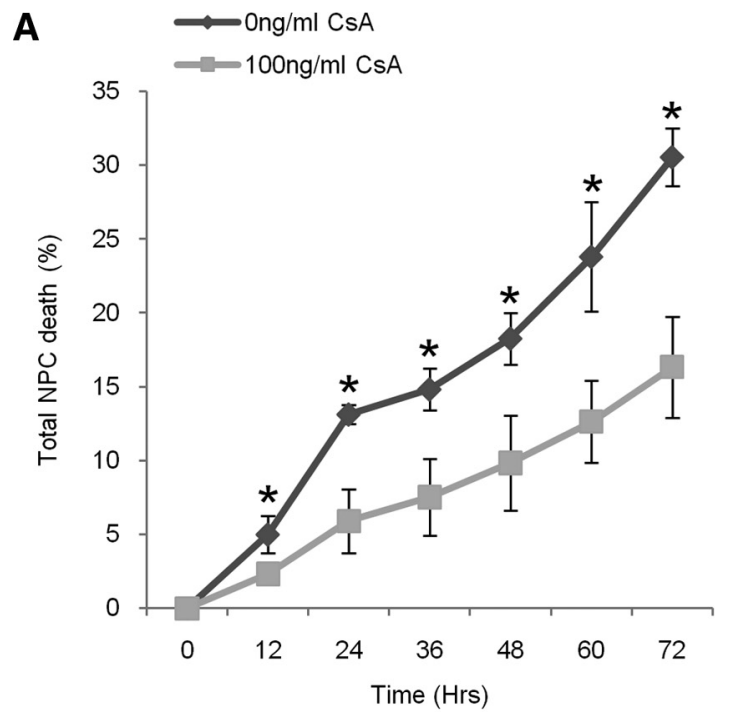

B

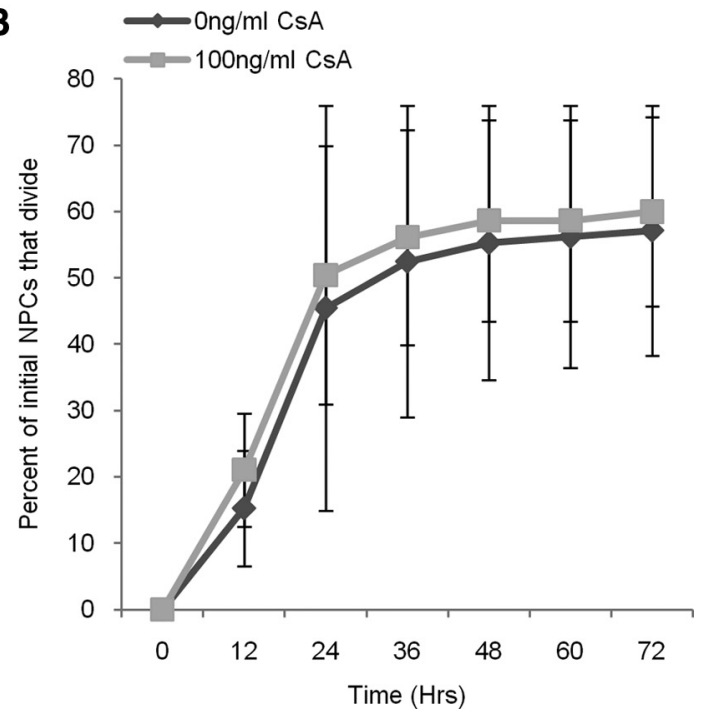

Figure 2. Cyclosporin A (CSA) enhanced NPC survival. $\boldsymbol{A}$, Significantly fewer cyclosporin A-treated NPCs die over $3 \mathrm{~d}$ compared with control NPCs $(p<0.05)$. $\boldsymbol{B}$, We observed no difference in the numbers of cyclosporin A-treated and control NPCs to undergo at least one division (data represents mean $\pm \mathrm{SEM}$ ).

with the untreated colonies. After $3 \mathrm{~d}$, the average size of cyclosporin A-treated colonies was more than twice that of control colonies [7 cells (range 1-20 cells) vs 3 cells (range 1-9 cells), respectively; $p<0.05$ ] (Fig. 3A). The increased colony size could be the result of a shorter cell cycle and/or enhanced cell survival within the developing clones. Indeed, even a small change in the time to the first cell division could have accounted for the doubling in colony size at $3 \mathrm{~d}$. The lineage trees generated from the developing clones revealed no significant increase in the rate of proliferation for cyclosporin A-treated NPCs, neither in the time to the first cell division $(19 \pm 4 \mathrm{~h}$ vs $21 \pm 7 \mathrm{~h}$, treatment vs control) nor in subsequent cell cycles $(21 \pm 1 \mathrm{~h}$ vs $23 \pm 0 \mathrm{~h}$, treatment vs control) (Fig. $3 B$ ). However, we did observe greater cell survival of NPCs within developing colonies treated with cyclosporin A. Lineage analysis revealed significantly less cell death within cyclosporin A-treated clones ( $15 \pm 3 \%$ vs $29 \pm 5 \%$, treatment vs control; $p<0.05$ ) (Fig. $3 C-F$ ) that could account for the larger colonies observed on day 3 . Hence, enhanced cell survival in the presence of cyclosporin A can account for both the increased numbers and size of the colonies in vitro.
Enhanced cell survival is not mediated by interleukin-2

Cyclophilin A is the major intracellular receptor for cyclosporin A. In mammals, the cyclosporin A-cyclophilin A complex binds to and inhibits calcineurin, which leads to inhibition of interleukin-2 production, a growth factor critical for T-cell proliferation. Given that cyclophilin A and calcineurin are abundantly expressed within the NPC population (Fig. 1A), we asked whether the effects on cell survival were mediated by cyclosporin A inhibiting interleukin-2 expression. We looked for the presence of interleukin-2 using RT-PCR from NPCs and splenic cells exposed to concanavalin A to induce interleukin-2 expression. As predicted, the concanavalin A-treated splenic cells expressed interleukin-2; however, concanavalin A-exposed NPCs were negative for interleukin-2 (Fig. 3G) as were untreated NPCs (data not shown). The lack of interleukin-2 expression from NPCs was confirmed using ELISA on conditioned medium from NPC cultures at 2, 4, or $7 \mathrm{~d}$ in vitro. At all times examined we were unable to detect interleukin-2 expression (data not shown). Hence, cyclosporin A enhances cell survival through a pathway independent of interleukin-2.

\section{Cyclosporin A does not alter the differentiation profile of multipotent colonies}

We asked whether the enhanced cell survival within developing colonies observed in the presence of cyclosporin A was selective for a particular cell type. We examined the differentiation profiles of cells derived from neurospheres as well as colonies formed in the gap chambers. At the population level, neurospheres were collected from cyclosporin A-treated and untreated cultures, plated on Matrigel-coated wells (1 sphere/well) in the presence of $10 \%$ FBS to induce cell differentiation for $7 \mathrm{~d}$. We observed no significant difference in the differentiation profile between control and treatment conditions. In both instances the vast majority of cells differentiated into GFAP-positive astrocytes ( $94 \pm 2 \%$ vs $93 \pm 2 \%$, treatment vs control), with fewer BIII/MAP-2-positive neurons $(3.5 \pm 1.1 \%$ vs $3.9 \pm 1.4 \%$, treatment vs control) and O4-positive oligodendrocytes $(2.2 \pm 1.1 \%$ vs $2.9 \pm 0.1 \%$, treatment vs control) (Fig. 4A). A small number of cells remained undifferentiated and stained positive for Nestin $(0.9 \pm 0.9 \%$ vs $0.7 \pm 0.4 \%$, treatment vs control).

Similar results were obtained from differentiated colonies within the gap chamber. NPCs in gap chambers were placed in growth factor conditions in the presence or absence of cyclosporin A for $4 \mathrm{~d}$ followed by the addition of $10 \%$ FBS on day 5 to induce cell differentiation, and fixation on day 8. In total, 108 untreated NPCs and 57 cyclosporin A-treated NPCs were imaged and tracked for $8 \mathrm{~d}$ from two independent trials. Consistent with what was observed in the undifferentiated $3 \mathrm{~d}$ colonies, the size of the cyclosporin A-treated colonies were significantly larger than control clones ( $16 \pm 2$ cells vs $7 \pm 5$ cells, treatment vs control; $p<0.05)$. We also observed more multipotent colonies in cyclosporin A-treated cultures ( $33 \%$ of colonies greater than three cells large vs $12 \%$ of colonies $>3$ cells large, treatment vs control). Similar to the differentiation profile from neurospheres, the relative frequency of neural cell types did not differ between the treated and control colonies whereby the majority of cells within multipotent clones were GFAP-positive astrocytes (67 $\pm 0 \%$ vs $63 \pm 4 \%$, treatment vs control) with a smaller fraction of BIII/ MAP-2-positive neurons ( $18 \pm 1 \%$ vs $21 \pm 4 \%$, treatment vs control) and $\mathrm{O} 4$-positive oligodendrocytes ( $15 \pm 2 \%$ vs $17 \pm 0 \%$, treatment vs control) (Fig. 4B). All cells within the multipotent clones stained positive for one of the neural lineages. The difference in the frequencies of neural cell types between neurospheres 
A

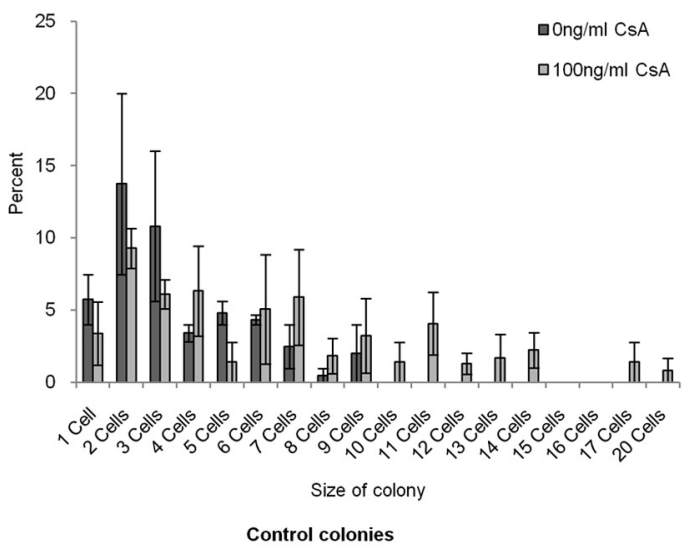

B

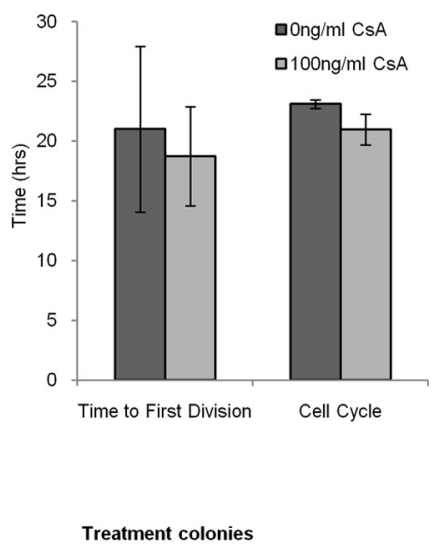

C
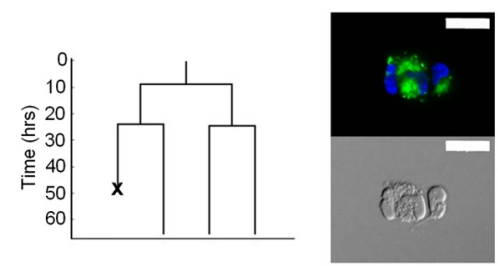

E

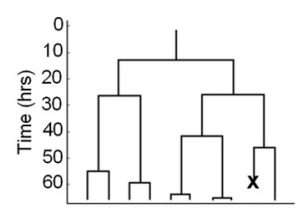

G

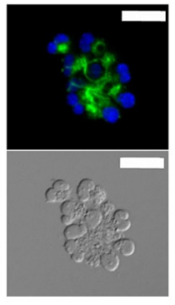

D

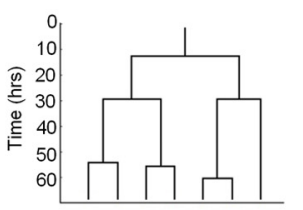

$F$

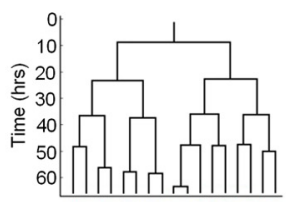

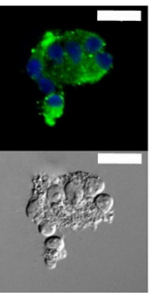

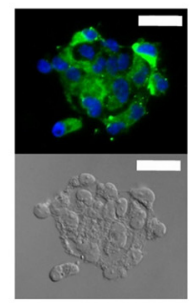

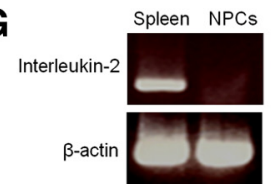

Figure 3. Cyclosporin A (CsA) increased the size of NPC colonies. $\boldsymbol{A}$, Cyclosporin A-treated clones were significantly larger than control clones $(p<0.05)$. $B$, The time to the first cell division and the average cell cycle did not differ between the control and treatment. However, lineage analysis revealed significantly less cell death occurred in cyclosporin A-treated clones and accounted for larger clones $(p<0.05)$. $\boldsymbol{C}-\boldsymbol{F}$, Lineage trees for the average control $(\boldsymbol{C})$, average treatment $(\boldsymbol{D})$, largest control $(\boldsymbol{E})$, and larger treatment $(\boldsymbol{F})$ clones. Lineage tree branches represent cell divisions, and " $X$ " $s$ within the lineage trees represent a cell death. All clones were stained for Nestin (green) and Hoechst (blue), and are pictured beside the corresponding lineage trees (scale bar, 20 $\mu \mathrm{m}) . \mathbf{G}$, Concanavalin A-treated NPCs do not produce interleukin-2 (data represent mean \pm SEM).

and colonies in gap chambers may be attributed to the different total lengths of time in culture and/or exposure to FBS and/or due to the differences in cell-cell contact during differentiation. While we cannot ascertain the reason for the difference in absolute numbers of neural phenotypes, the finding that the differentiation profiles are identical between treated and control colonies within the same paradigm (gap chamber or suspension culture) indicates that cyclosporin $\mathrm{A}$ is not promoting the selective cell proliferation and/or survival of particular lineages within developing clones.

\section{Cyclosporin A modifies cell-cell adhesions increasing the numbers of clones}

During colony formation, NPCs are extremely adherent to one another and only rarely do cells separate from their developing colony (Ramunas et al., 2006) (supplemental Movie S1, available at www.jneurosci.org as supplemental material). Importantly, the rare instances of cell separation in the control conditions always resulted in death of the departing cell. In sharp contrast, we frequently observed cells separating from their colony of origin in cyclosporin A conditions. In cyclosporin A conditions, $21 \pm 11 \%$ of dividing NPCs had at least one daughter cell leave the parent colony versus $3 \pm 3 \%$ in the control cultures (see supplemental Movies S2 and S3, available at www. jneurosci.org as supplemental material) (Fig. $5 A, B)(p<0.05)$. Further, virtually all of the departing NPCs survived over the same time period in the presence of cyclosporin $A$ and either returned to their colony of origin $(25 \%$ of the departing cells) or remained separate, thereby generating multiple colonies ( $75 \%$ of the departing cells). Indeed, the majority of departing cells in cyclosporin A continued to proliferate following separation (76 \pm $14 \%$ ) forming colonies that were $2-5$ cells in size. Morphological differences were also observed whereby cyclosporin A-treated NPCs extended multiple long cell processes before and after separation (supplemental Movies S2 and S3, available at www.jneurosci.org as supplemental material). Hence, concomitant with increased cell survival, cyclosporin A treatment decreases cell-cell adhesions which may account, in part, for the observed increase in the numbers of cyclosporin A-treated colonies. Interestingly, extracellular cyclophilin A and B have been shown to influence cell adhesion by binding a type I integral membrane receptor, Cd147, which in turn binds Cd98 (a glycoprotein) and causes a cascade of events leading to matrix metalloproteinase production, cell adhesion and attachment, and cytoskeletal rearrangement (Allain et al., 1999; Yurchenko et al., 2002; Jin et al., 2004; Seko et al., 2004). We performed PCR for Cd147 and Cd98 and found them to be present on neurosphere-derived cells (Fig. 5C) suggesting that these molecules may be playing a role in the modified cell-cell adhesion observed between the NPCs.

\section{Cyclosporin A affects NPCs in vivo}

We next asked whether cyclosporin A exposure in vivo would affect NPCs behavior. Adult animals received $14 \mathrm{~d}$ infusions of cyclosporin A ( $15 \mathrm{mg} / \mathrm{kg} / \mathrm{d})$ or saline (control) delivered via subcutaneously implanted osmotic mini-pumps. Neurosphere cultures were established and the numbers of neurospheres were counted after $7 \mathrm{~d}$. We observed a significant 2.6-fold increase in the numbers of neurospheres derived from cyclosporin A-treated animals ( $31 \pm 3$ spheres vs $12 \pm 3$ spheres, treatment vs control, $p<0.01)$ consistent with our in vitro findings that cyclosporin A enhances neural stem cell survival. Intraventricular infusion of cyclosporin A also resulted in a significant twofold increase in numbers of neurospheres ( $12 \pm 1$ spheres vs $6 \pm 0.4$ spheres, treatment vs control, $n=4, p<0.01)$. The average diameter of 
neurospheres did not differ between groups indicating connection between in vitro cyclosporin A exposure and increased NPC survival $(121 \pm 4 \mu \mathrm{m}$ vs $117 \pm 1 \mu \mathrm{m}$, treatment vs control). To ask whether the increased numbers of neural stem cells in vivo translated into increased numbers of neural stem cell progeny, a separate group of animals received a series of BrdU injections on the day of death to label the proliferating stem cell progeny. Cyclosporin A-treated animals had a significant 1.5 -fold increase in the numbers of BrdU labeled cells in the subependyma lining the lateral ventricles $(n=4, p<$ 0.01 ). Together, these data are consistent with cyclosporin A enhancing NPC survival and reveal that cyclosporin $\mathrm{A}$ can modify NPC behavior both in vitro and in vivo.

\section{Discussion}

In this study, we have examined the behavior of neural stem and progenitor cells in response to cyclosporin A using both in vitro and in vivo models. Using pure populations of NPCs we established that cyclosporin A has direct effects on NPCs; specifically enhancing cell survival and decreasing cell-cell adhesion. Moreover, the direct effects of cyclosporin A on NPC survival are independent of the interleukin-2 pathway. The enhanced cell survival was not selective for a particular lineage as the differentiation profiles of the cells were similar in the presence or absence of cyclosporin A. Interestingly, we found that cyclosporin A administration to uninjured animals could increase the numbers of neural stem cells and their progeny, which suggests that intracellular targets of cyclosporin A may provide novel therapeutic targets for acting on endogenous progenitor cells in the development of regenerative strategies.

There are multiple intracellular pathways that cyclosporin A can play a role in through binding with specific cyclophilins. For instance, previous studies have reported neuroprotective effects of cyclosporin A following CNS injury (Shiga et al., 1992; Uchino et al., 1995). One of the best characterized mechanisms by which cyclosporin A is thought to enhance cell survival is through inhibition of the mitochondrial permeability transition (MPT) pore formation. Permeability transition is a sudden increase in the inner mitochondrial membrane permeability to ions and solutes through the MPT pore causing dissipation of mitochondrial membrane potential, loss of mitochondrial ion homeostasis, impairment of ATP synthesis, mitochondrial swelling, outer membrane rupture, and ultimately cell death (Basso et al., 2005). Cyclophilin D is required for MPT pore formation and cyclosporin A is known to bind and block cyclophilin D's actions, thus preventing cell death (Baines et al., 2005). Cyclosporin A may also prevent cell death by inhibiting the action of calcineurin, preventing the upregulation of nitric oxide synthase and thus blocking production of nitric oxide and reactive oxygen species (Snyder et al., 1998). It has also been proposed that blocking mean \pm SEM.
B

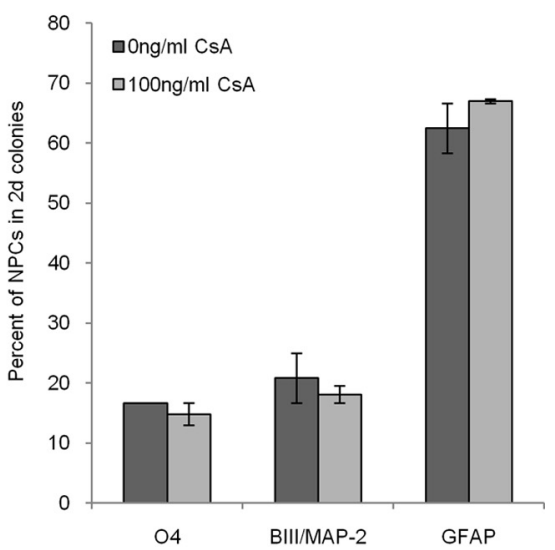

G

E

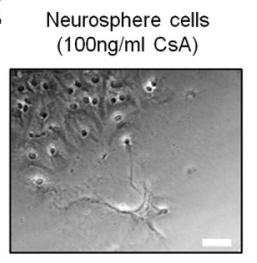

$\mathbf{F}$

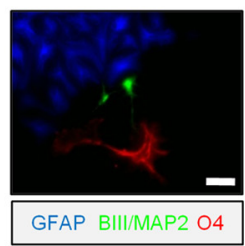

H
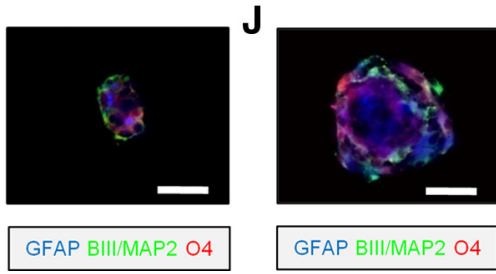

GFAP BIII/MAP2 O4

Figure 4. Cyclosporin A (CSA) did not promote selective cell survival. $A, B$, The percentages of oligodendrocytes, neurons, and astrocytes within neurospheres $(\boldsymbol{A})$ and within multipotent gap chamber NPC colonies ( $2 \mathrm{~d}$ cell colonies) $(\boldsymbol{B})$ did not differ between the control and treatment. $\boldsymbol{C}-\boldsymbol{J}$, Examples of control neurospheres $(\boldsymbol{C}, \boldsymbol{D})$, treatment neurospheres $(\boldsymbol{E}, \boldsymbol{F})$, control $2 \mathrm{~d}$ colonies $(\boldsymbol{G}, \boldsymbol{H})$, and treatment $2 \mathrm{~d}$ colonies $(\boldsymbol{I}, \boldsymbol{J})$. Red, Oligodendrocytes; green, neurons; blue, astrocytes. Scale bar, $50 \mu \mathrm{m}$. Data represent

calcineurin directly affects cell viability by inhibiting Bad dephosphorylation, which in turn prevents Bad from entering the mitochondria and blocking the prosurvival protein $B c l-x L$ (Kaminska et al., 2004). Hence, in addition to the well described immunosuppressive effects of cyclosporin A, the prosurvival effects mediated by various cyclophilins have been documented.

The roles for cyclosporin A in cell adhesion have previously been documented with immune cells both in vitro and in vivo. Cyclophilins $A$ and $B$ were shown to be released into extracellular space at high levels in response to inflammatory stimuli including severe sepsis (Tegeder et al., 1997), HIV infection (Endrich and Gehring, 1998), and oxidative stress (Jin et al., 2000). Cyclophilin A induces chemotaxis of eosinophils, neutrophils, and T cells and extracellular cyclophilin B induces chemotaxis and adhesion to the extracellular matrix of memory CD4 ${ }^{+}$T cells (Sherry et al., 1992; Xu et al., 1992; Allain et al., 1999). CD147, the receptor for extracellular cyclophilins A and B, stimulates phosphorylation of ERK1/2, p38, JNK, Akt, and IkB inducing matrix metalloproteinase production, cell adhesion, HIV attachment, and cytoskeletal rearrangement (Jin et al., 2004; Seko et al., 2004; Yurchenko et al., 2005). CD147 also binds to CD98 resulting in changes of cytoskeletal elements as well as cell-cell adhesions; however, the precise mechanism of action is currently unknown. Interestingly, our preliminary results show CD147 and CD98 are present in NPC populations suggesting that this mechanism may also play a role in the cell adhesion between NPCs. 
A

Treatment: original clone, subclone $a$, subclone $b$, subclone $\mathrm{c}$, subclone $\mathrm{d}$

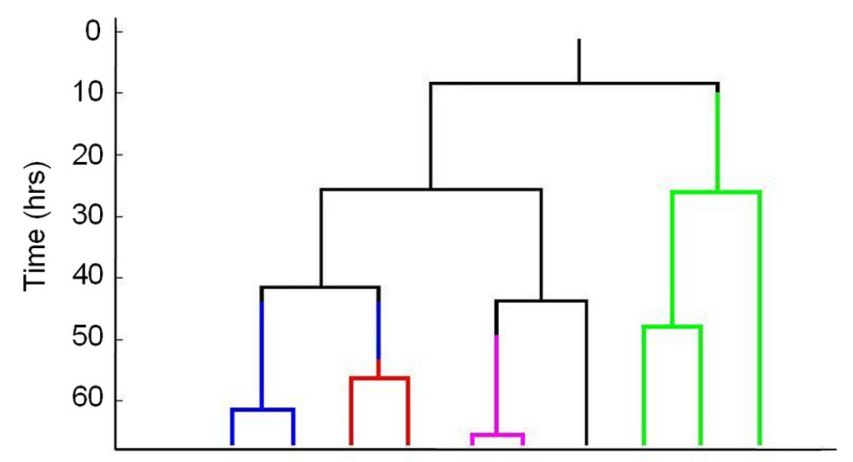

B

Control: original clone, subclone a

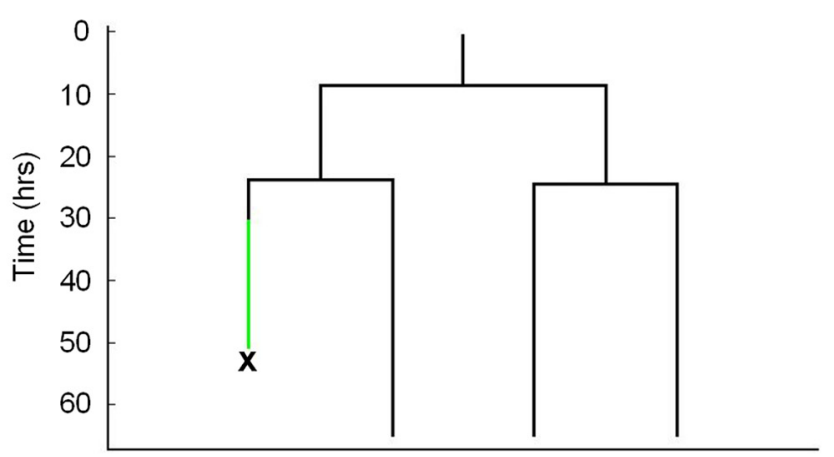

C

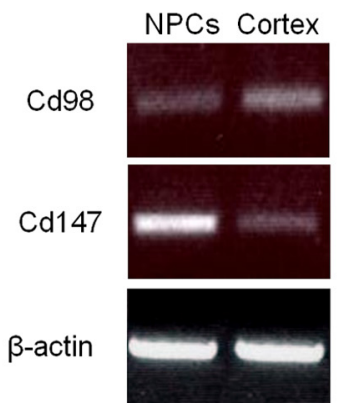

Figure 5. Cyclosporin A decreased cell-cell adhesions. $\boldsymbol{A}$, Multiple separations in a cyclosporin A-treated colony resulted in five colonies forming from one NPC. Each different color in the lineage tree represents a cell separation and new NPC subclone. $\boldsymbol{B}$, In a control clone, one NPC separates from a clone that is four cells large, but the cell dies following separation. C, RTPCR confirmed the presence of $\mathrm{Cd} 98$ and Cd147 in P1 neurosphere-derived cells (data represent mean \pm SEM).

The effects of cyclosporin A have more recently been examined using hippocampal precursor cells from embryonic animals. In contrast to our results, Guo et al. (2007) reported decreased proliferation of embryonic hippocampal-derived precursors in vitro in the presence of cyclosporin A as well as decreased numbers of neurons and increased numbers of astrocytes within cyclosporin A-treated colonies. The differences between these studies and the current investigation likely reflect the use of temporally (embryonic vs adult) and regionally distinct (hippocampal vs subependymal) precursor pools examined highlighting the differences between starting populations of cells.

Consistent with our in vitro findings, cyclosporin A infusion into animals also increases neurosphere numbers and a concomitant increase in the numbers of BrdU-labeled subependymal cells. We cannot directly rule out the possibility that the increased BrdU labeling is due to an effect of cyclosporin A on cell proliferation; based on our in vitro findings showing no change in the proliferation kinetics of NPCs in the presence of cyclosporin A, we feel that it is fair to conclude that the effects are the result of enhanced cell survival. Factors that affect the survival and/or mode of division of stem cells are of interest for stem cell biology in general. Stem cells in general can divide symmetrically (to produce two stem cell daughters) thereby expanding the pool of precursors or asymmetrically (to self-renew and generate one precursor cell) and maintain the size of the precursor population. Previous studies on subependymal cell proliferation kinetics suggest that, under baseline conditions, neural stem cells divide asymmetrically in vivo (Morshead et al., 1998). However, if neural stem cells divide symmetrically in vivo and one neural stem cell daughter dies, cyclosporin A may enhance the survival of the stem cell that normally dies. This could account for the increased numbers of neurospheres derived from cyclosporin A-treated animals. Notably, while the in vivo observations are consistent with the in vitro findings, the increase in neurospheres from uninjured animals following cyclosporin A treatment could reflect an indirect action of cyclosporin A on NPCs.

In a recent study, Louis et al. (2008) reported the development of the neural colony-forming cell assay (NCFCA) which involved the use of a semisolid medium to grow neural-derived colonies for a period of $21 \mathrm{~d}$, which ensures clonality and discriminates neural stem from progenitor cells on the basis of their proliferative potential. The report suggests that the NCFCA can distinguish between progenitor-derived and neural stem cell-derived colonies based on the size of the colony after $21 \mathrm{~d}$ in culture. Our findings are entirely consistent with their observation since virtually all the large clones ( $>100 \mu \mathrm{m}$ neurospheres) that we take after $7 \mathrm{~d}$ in suspension culture are multipotent. Interestingly, in our study we observed a significant increase in the numbers of large neurospheres after $7 \mathrm{~d}$ in vitro, consistent with the Louis et al. (2008) report that large colonies are derived from stem cells. Further, recent work in our lab has demonstrated that the vast majority $(>95 \%)$ of the neurospheres $>100 \mu \mathrm{m}$ in diameter at $7 \mathrm{~d}$ in suspension culture will continue to increase in size over the $21 \mathrm{~d}$ culture period, similar to what is observed in the NCFCA. In regard to ensuring clonality, the NCFCA assay does not have an advantage over low-density suspension culture (as we used in our study), for which we have demonstrated that clonality can be preserved when the cultures are not moved during the $7 \mathrm{~d}$ in vitro (Coles-Takabe et al., 2008). Hence, this continued proliferative capacity of the $>100 \mu \mathrm{m}$ neurospheres, combined with the observation that $100 \%$ can be single sphere passaged and are multipotent upon differentiation, supports our statement that the numbers of neurospheres reflects the numbers of stem cells.

Cyclosporin A's effect on neural stem and progenitor cells in vivo is of particular interest when considering the development of strategies that target endogenous NPCs and/or transplant NPCs to promote repair. Injury has been shown to increase proliferation and migration of endogenous NPCs although the response is quite limited in magnitude and duration (Arvidsson et al., 2002; Zhang et al., 2004; Kolb et al., 2007). Hence, increasing the size of the NPC pool available to contribute to tissue regeneration is a promising goal. It is also interesting to consider interactions between grafted NPCs and endogenous NPCs in cyclosporin A immunosuppressed animals. Cyclosporin A is often used in studies to inhibit graft rejection and given our findings, the possibility remains that some of the effects attributed to transplanted cells may be due to enhanced survival of grafted NPCs and/or due to 
the activation of endogenous precursors. We have shown, for the first time, a direct effect of cyclosporin A on NPCs in vitro and in vivo indicating cyclosporin $\mathrm{A}$ is an excellent candidate molecule for future studies.

\section{References}

Allain F, Durieux S, Denys A, Carpentier M, Spik G (1999) Cyclophilin B binding to platelets supports calcium dependent adhesion to collagen. Blood 94:976-983.

Arvidsson A, Collin T, Kirik D, Kokaia Z, Lindvall O (2002) Neuronal replacement from endogenous precursors in the adult brain after stroke. Nat Med 8:963-970.

Baines CP, Kaiser RA, Purcell NH, Blair NS, Osinska H, Hambleton MA, Brunskill EW, Sayen MR, Gottlieb RA, Dorn GW, Robbins J, Molkentin JD (2005) Loss of cyclophilin D reveals a critical role for mitochondrial permeability transition in cell death. Nature 434:658-662.

Basso E, Fante L, Fowlkes J, Petronilli V, Fortes MA, Bernardi P (2005) Properties of the permeability transition pore in mitochondria devoid of Cyclophilin D. J Biol Chem 19:18558-18561.

Ben-Hur T, Ben-Menachem O, Furer V, Einstein O, Mizrachi-Kol R, Grigoriadis N (2003) Effects of proinflammatory cytokines on the growth, fate, and motility of multipotential neural precursor cells. Mol Cell Neurosci 24:623-631.

Ben-Hur T, Ben-Yosef Y, Mizrachi-Kol R, Ben-Menachem O, Miller A (2006) Cytokine-mediated modulation of MMPs and TIMPs in multipotential neural precursor cells. J Neuroimmunol 175:12-18.

Borel JF, Feuer C, Gubler HU, Steahelin H (1976) Biological effects of cyclosporine A: a new antilymphocytic agent. Agents Actions 6:465-468.

Butovsky O, Ziv Y, Schwartz A, Landa G, Talpalar AE, Pluchino S, Martino G, Schwartz M (2006) Microglia activated by IL-4 or IFN-gamma differentially induce neurogenesis and oligodendrogenesis from adult stem/progenitor cells. Mol Cell Neurosci 31:149-160.

Coles-Takabe BLK, Brain I, Purpura KA, Karpowicz P, Zandstra PW, Morshead CM, van der Kooy D (2008) Don't look: growing clonal versus nonclonal neural stem cell colonies. Stem Cells 26:2938-2944.

Dawson TM, Steiner JP, Lyons WE, Fotuhi M, Blue M, Snyder SH (1994) The immunophilins, FK506 binding protein and cyclophilin, are discretely localized in the brain: relationship to calcineurin. Neuroscience 62:569-580.

Endrich M, Gehring H (1998) The V3 loop of human immunodeficiency virus type- 1 envelope protein is a high-affinity ligand for immunophilins present in human blood. Eur J Biochem 252:441-446.

Erlandsson A, Morshead CM (2006) Exploiting the properties of adult neural stem cells for the treatment of disease. Curr Opin Mol Ther 8:331-337.

Fischer G, Wittmann-Liebold B, Lang K, Kiefhaber T, Schmid FX (1989) Cyclophilin and peptidyl-prolyl cis-trans isomerase are probably identical proteins. Nature 337:476-478.

Flanagan WM, Corthésy B, Bram RJ, Crabtree GR (1991) Nuclear association of a T-cell transcription factor blocked by FK-506 and cyclosporin A. Nature 352:803-807.

Fruman DA, Klee CB, Bierer BE, Burakoff SJ (1992) Calcineurin phosphatase activity in T lymphocytes is inhibited by FK-506 and cyclosporin A. Proc Natl Acad Sci U S A 89:3686-3690.

Guo J, Zeng Y, Liang Y, Wang L, Su H, Wu W (2007) Cyclosporine affects the proliferation and differentiation of neural stem cells in culture. Neuroreport 18:863-868.

Guo X, Dillman JF, Dawson VL, Dawson TM (2001) Neuroimmunophilins: novel neuroprotective and neuroregenerative targets. Ann Neurol 50:6-16.

Hamilton GS, Steiner JP (1998) Immunophilins: beyond immunosuppression. J Med Chem 41:5119-5143.

Handschumacher RE, Harding MW, Rice J, Drugge RJ, Speicher DW (1984) Cyclophilin: a specific cytosolic binding protein for cyclosporin A. Science 226:544-547.

Jin ZG, Melaragno MG, Liao DF, Yan C, Haendeler J, Suh YA, Lambeth D, Berk BC (2000) Cyclophilin A is a secreted growth factor induced by oxidative stress. Circ Res 87:789-796.

Jin ZG, Lungu AO, Xie L, Wang M, Wong C, Berk BC (2004) Cyclophilin A is a proinflammatory cytokine that activates endothelial cells. Arterioscler Thromb Vasc Biol 24:1186-1191.

Kaminska B, Gaweda-Walerych K, Zawadzka M (2004) Molecular mechanisms of neuroprotective action of immunosuppressants-facts and hypotheses. J Mol Med 8:45-58.
Kay JE, Benzie CR, Borghetti AF (1983) Effect of cyclosporine A on lymphocyte activation by the calcium ionophore A23187. Immunology 50:441446.

Kolb B, Morshead C, Gonzalez C, Kim M, Gregg C, Shingo T, Weiss S (2007) Growth factor-stimulated generation of new cortical tissue and functional recovery after stroke damage to the motor cortex of rats. J Cereb Blood Flow Metab 27:983-997.

Liu J, Farmer JD Jr, Lane WS, Friedman J, Weissman I, Schreiber SL (1991) Calcineurin is a common target of cyclophilin-cyclosporin A and FKBPFK506 complexes. Cell 66:807-815.

Lois C, Alvarez-Buylla A (1994) Long-distance neuronal migration in the adult mammalian brain. Science 264:1145-1148.

Louis SA, Rietze RL, Deleyrolle L, Wagey RE, Thomas TE, Eaves AC, Reynolds BA (2008) Enumeration of neural stem and progenitor cells in the neural colony-forming cell assay. Stem Cells 26:988-996.

Morshead CM, Reynolds BA, Craig CG, McBurney MW, Staines WA, Morassutti D, Weiss S, van der Kooy D (1994) Neural stem cells in the adult mammalian forebrain: a relatively quiescent subpopulation of subependymal cells. Neuron 13:1071-1082.

Morshead CM, Craig CG, van der Kooy D (1998) In vivo clonal analyses reveal the properties of endogenous neural stem cell proliferation in the adult mammalian forebrain. Development 125:2251-2261.

Morshead CM, Benveniste P, Iscove NN, van der Kooy D (2002) Hematopoietic competence is a rare property of neural stem cells that may depend on genetic and epigenetic alterations. Nat Med 8:268-273.

Morshead CM, Garcia AD, Sofroniew MV, van der Kooy D (2003) The ablation of glial fibrillary acidic protein-positive cells from the adult central nervous system results in the loss of forebrain neural stem cells but not retinal stem cells. Eur J Neurosci 18:76-84.

Pluchino S, Martino G (2008) Neural stem cell-mediated immunomodulation: repairing the haemorrhagic brain. Brain 131:604-605.

Ramunas J, Illman M, Kam A, Farn K, Kelly L, Morshead CM, Jervis EJ (2006) True monolayer cell culture in a confined 3D microenvironment enables lineage informatics. Cytometry 69:1202-1211.

Reynolds BA, Weiss S (1992) Generation of neurons and astrocytes from isolated cells of the adult mammalian central nervous system. Science 255:1707-1710.

Seko Y, Fujimura T, Taka H, Mineki R, Murayama K, Nagai R (2004) Hypoxia followed by reoxygenation induces secretion of cyclophilin A from cultured rat cardiac myocytes. Biochem Biophys Res Commun 317:162168 .

Sherry B, Yarlett N, Strupp A, Cerami A (1992) Identification of cyclophilin as a proinflammatory secretory product of lipopolysaccharide-activated macrophages. Proc Natl Acad Sci U S A 89:3511-3515.

Shiga Y, Onodera H, Matsuo Y, Kogure K (1992) Cyclosporin A protects against ischemia-reperfusion injury in the brain. Brain Res 595:145-148.

Snyder SH, Lai MM, Burnett PE (1998) Immunophilins in the nervous system. Neuron 21:283-294.

Takahashi N, Hayano T, Suzuki M (1989) Peptidyl-prolyl cis-trans isomerase is the cyclosporin A-binding protein cyclophilin. Nature 337:473475.

Tegeder I, Schumacher A, John S, Geiger H, Geisslinger G, Bang H, Brune K (1997) Elevated serum cyclophilin levels in patients with severe sepsis. J Clin Immunol 17:380-386.

Uchino H, Elmér E, Uchino K, Lindvall O, Siesjö BK (1995) Cyclosporin A dramatically. ameliorates CA1 hippocampal damage following transient forebrain ischaemia in the rat. Acta Physiol Scand 155:469-471.

Wang P, Heitman J (2005) The cyclophilins. Genome Biol 6:226-231.

Xu Q, Leiva MC, Fischkoff SA, Handschumacher RE, Lyttle CR (1992) Leukocyte chemotactic activity of cyclophilin. J Biol Chem 267:11968-11971.

Yurchenko V, Zybarth G, O'Connor M, Dai WW, Franchin G, Hao T, Guo H, Hung HC, Toole B, Gallay P, Sherry B, Bukrinsky M (2002) Active site residues of Cyclophilin A are crucial for its signaling activity via CD147. J Biol Chem 277:22959-22965.

Yurchenko V, Constant S, Bukrinsky M (2006) Dealing with the family: CD147 interactions with cyclophilins. Immunology 117:301-309.

Zhang R, Zhang Z, Zhang C, Zhang L, Robin A, Wang Y, Lu M, Chopp M (2004) Stroke transiently increases subventricular zone cell division from asymmetric to symmetric and increases neuronal differentiation in the adult rate. J Neurosci 24:5810-5815. 